\title{
ARTICLE \\ Intrinsic differences in insular circuits moderate the negative association between nicotine dependence and cingulate-striatal connectivity strength
}

\author{
Robin J. Keeley $\mathbb{I D}^{1}$, Li-Ming Hsu iD $^{1,2}$, Julia K. Brynildsen ${ }^{1,3}$, Hanbing Lu' ${ }^{1}$ Yihong Yang ${ }^{1}$ and Elliot A. Stein ${ }^{1}$
}

\begin{abstract}
The development of brain-based biomarkers to assess nicotine dependence severity and treatment efficacy are essential to improve the current marginally effective treatment outcomes. Cross-sectional resting state functional connectivity (rsFC) studies in humans identified a circuit between the dorsal anterior cingulate cortex and the ventral striatum that negatively correlated with increased nicotine dependence severity but was unaffected by acute nicotine administration, suggesting a trait marker of addiction. However, whether this trait circuit dysregulation is predispositional to or resultant from nicotine dependence is unclear. Using a rat model of nicotine dependence with longitudinal fMRI measurements, we assessed the relationship between ACC-striatal rsFC and nicotine dependence severity. Data-driven modularity-based parcellation of the rat medial prefrontal cortex (mPFC) combined with seed-based connectivity analysis with the striatum recapitulated the cingulate-striatum relationship observed in humans.

Furthermore, the relationship between cingulate-striatal brain circuits and nicotine dependence severity as indexed by the intensity of precipitated withdrawal, was fully statistically moderated by a predispositional insular-frontal cortical functional circuit. These data suggest that the identified trans-species ACC-striatal circuit relationship with nicotine dependence severity is dysregulated following chronic nicotine administration-induced dependence and may be biased by individual differences in predispositional insula-based striatal-frontal circuits, highlighting the circuit's potential as a biomarker of dependence severity.
\end{abstract}

Neuropsychopharmacology (2020) 45:1042-1049; https://doi.org/10.1038/s41386-020-0635-x

\section{INTRODUCTION}

Smoking is the leading cause of preventable death in the US [1]. Current behavioral and pharmacological treatment strategies are only marginally effective, with $75-95 \%$ of smokers relapsing within 6 months of a quit attempt [2]. Incomplete understanding of nicotine addiction as a system level brain disease necessitates the establishment of brain-based biomarkers of disease severity, which would permit the assessment of novel, individually targeted treatment strategies and more efficacious treatments for smoking and resultant nicotine addiction [3]. Cross-sectional studies in smokers using functional magnetic resonance imaging (fMRI) resting state functional connectivity ( $\mathrm{rSFC}$ ) have identified that connectivity between the dorsal anterior cingulate cortex (dACC) and the ventral striatum (VS) is negatively associated with nicotine dependence severity [4]. The strength of this functional circuit, which is modulated by genetic polymorphisms in the a5 nicotinic receptor [5] and the nicotine metabolism enzyme CYP2A6 [6], is unchanged following acute nicotine patch administration, suggesting its utility as a putative biomarker of trait nicotine addiction. Although these regions and circuits were recapitulated in these three studies from independent cohorts of smokers using distinct analytical strategies, it remains unknown whether they were predispositional and contributed to the development of dependence or were resultant from long-term smoking.

fMRI provides unique translational and reverse translational systems level insight, since measures obtained across species are based on a common signal from the same biophysical principles [7]. Preclinical fMRI is uniquely suited to query the resultant and/or predispositional nature of brain circuits, and consistent homologies in brain function and organization have been identified $[8,9]$. Using this approach, we have recently identified insular centric circuits in a rat model of nicotine dependence that predisposed to subsequent nicotine dependence severity [10]. Thus, to mechanistically explain the relationship between ACCstriatal rsFC circuit alterations and nicotine dependence severity observed in humans, we combined longitudinal fMRI and behavioral pharmacology in drug naive and nicotine-dependent rats to expand upon our previous predispositional circuit findings [10] and uncover the relationship between predispositional circuits and those that change as a function of nicotine dependence.

Although there is general agreement as to the anatomical constituents of the rat medial prefrontal cortex (mPFC), which includes the infralimbic (IL), prelimbic ( $\mathrm{PrL}$ ) and cingulate cortices

\footnotetext{
${ }^{1}$ Neuroimaging Research Branch, National Institute on Drug Abuse, Intramural Research Program, 251 Bayview Blvd, Baltimore, MD 21224 , USA Correspondence: Elliot A. Stein (estein@nih.gov)

${ }^{2}$ Present address: Department of Radiology and BRIC, University of North Carolina at Chapel Hill, 130 Mason Farm Rd, Chapel Hill, NC 27599, USA

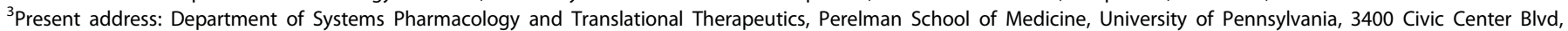
Philadelphia, PA 19104, USA

These authors contributed equally: R. J. Keeley, L.-M. Hsu
}

Received: 24 September 2019 Revised: 17 January 2020 Accepted: 4 February 2020

Published online: 13 February 2020 
[11-13], there is little consensus on the functional and/or anatomical homology to the human ACC $[14,15]$. We thus first applied data driven, graph theory based modularity analysis [16] to segment the rat mPFC into modules or regions with similar functional connectivity patterns. Rodent models of nicotine dependence [17] show dose- and duration-dependent withdrawal signs [18] similar to that observed in humans [19]. As withdrawal severity in humans predicts smoking relapse $[20,21]$, we administered mecamylamine, a nonspecific nicotinic receptor antagonist, to precipitate somatic withdrawal and used a quantitative measure of behavioral signs as a marker of nicotine dependence severity [22]. We next quantified functional circuits between these MPFC modules and the striatum and assessed the relationship of identified circuits with nicotine dependence severity. We predicted that a similar negative association between cingulate-striatal connectivity and nicotine dependence severity would be observed, paralleling that observed in humans. Finally, we performed moderation analyses to determine the role of individual differences in pre-drug insular-based circuits [10] on the relationship between nicotine-induced brain circuit changes and nicotine dependence. Using this exploratory model, we further predicted that pre-drug baseline connectivity would moderate the observed connectivity-dependence relationship.

\section{MATERIALS AND METHODS}

Subjects and general experimental design

Groups of male Sprague-Dawley rats (275-300 g; $n=12$ /group) were administered saline, low $(1.2 \mathrm{mg} / \mathrm{kg} / \mathrm{d})$ or high dose nicotine $(4.8 \mathrm{mg} / \mathrm{kg} / \mathrm{d})$ with nicotine doses calculated as free base, using an implanted osmotic minipump that passively delivered intermittent ( $1 \mathrm{~h}$ ON-1 h OFF) nicotine for $\sim 14$ days [17]. The experimental design was based on a previous behavioral study [17]; imaging results from the current cohort of rats, which addressed a different but complementary question, have been published previously [10]. Only male rats were used in this study given the known sex differences in response to nicotine, particularly in relation to nicotine withdrawal [for review, see 23]. Somatic signs of precipitated nicotine withdrawal, used as an index of nicotine dependence severity, were induced via s.c. injection of $1.5 \mathrm{mg} / \mathrm{kg}$ mecamylamine [22] and were assessed one day before each MRI scan: Day 0 (pre-drug baseline); Day 15 (peak dependence) and Day 29 (after 14 days of abstinence). MRI scanning was performed before pump implantation on Day 1 (pre-drug baseline), 16 (peak dependence) and 30 (abstinence). See Fig. 1a for experimental timeline. Imaging was conducted on separate days from precipitated withdrawal testing to ensure rats were not in acute, precipitated withdrawal during scanning. Investigators were blind to group during behavioral testing and MRI acquisition. Data from a subset of rats (pre-drug: $n=4$; peak dependence: $n=1$; abstinence: $n=3$ ) were not analyzed due to insufficient anesthesia plane, with the final N's for each day as follows: pre-drug: SAL: $n=11, \mathrm{LN}: n=11, \mathrm{HN}: n=10$; peak dependence: SAL: $n=11, \mathrm{LN}$ : $n=12, \mathrm{HN}: n=12$; abstinence: SAL: $n=12, \mathrm{LN}: n=11, \mathrm{HN}: n=$ 10). All study procedures were approved by the Animal Care and Use Committee of the National Institute on Drug Abuse, Intramural Research Program.

Intermittent nicotine administration

An intermittent, passive nicotine administration approach, developed by our group [17], used a modified osmotic minipump (model 2ML4; Alzet) in combination with a Lynch coil (PE60 tubing) to deliver i.p. saline or nicotine (nicotine hydrogen tartrate salt, dissolved in $0.9 \%$ sodium chloride and $\mathrm{pH}$ adjusted to $7.2+$ 0.5 ) in a fixed interval $1 \mathrm{~h}-\mathrm{ON}-1 \mathrm{~h}-\mathrm{OFF}$ pattern for $\sim 14$ days, starting on Day 1. This method of intermittent nicotine delivery produces consistent and precise phasic delivery of nicotine and induces heightened and persistent dose-dependent precipitated withdrawal behaviors as compared to continuous minipump delivery [17]. Nicotine doses of 1.2 and $4.8 \mathrm{mg} / \mathrm{kg} / \mathrm{d}$ were chosen based upon previous studies of nicotine dependence [22, 24-26]. Pumps were implanted under isoflurane (2-3\%) anesthesia.

\section{MRI acquisition and preprocessing}

Rats were anesthetized using a combination of low-dose isoflurane $(0.25-0.5 \%)$ and dexmedetomidine (s.c., $0.015 \mathrm{mg} / \mathrm{kg} / \mathrm{h}$ ) during MRI scanning using a Bruker Biospin $9.4 \mathrm{~T} / 30 \mathrm{~cm}$ scanner, as previously described [27]. BOLD acquisition began at least $1 \mathrm{~h}$ after anesthesia induction to allow monitored physiology (heart rate, blood pressure, respiration and body temperature) to stabilize [27, 28]. Physiological parameters did not vary as a function of nicotine dose nor was there a dose $x$ day interaction on any monitored physiological measure (Fig. S1). At the beginning of each scan session, a RARE sequence was used to acquire high-resolution, T2weighted anatomical images, which were subsequently used for regional anatomical localization. A gradient-echo EPI sequence $($ FOV $=32 \mathrm{~mm}$, matrix size $=64 \times 64$, repetition time $=1 \mathrm{~s}$, echo time $=15 \mathrm{~ms}, 15$ slices of $1 \mathrm{~mm}$ thickness) was used to acquire an fMRI dataset of 900 volumes.

Using AFNI [29], preprocessing steps included discarding the first 10 volumes to allow the MRI signal to reach steady state, slice timing correction, motion correction and spatial smoothing $0.6 \mathrm{~mm}$ full-width at half-maximum). The $\mathrm{fMRI}$ images were aligned to their corresponding T2-weighted images and then normalized to a common 3D space aligned with a rat stereotaxic atlas [30]. To improve the accuracy of spatial normalization, fMRI data from each rat were aligned to the group averaged images. Finally, the normalized images were linearly detrended and band-pass filtered $(0.01<\mathrm{f}<0.5 \mathrm{~Hz})$. This band-pass filter was chosen to retain high frequency power (i.e., $>0.1 \mathrm{~Hz}$ ) and to remove noise generated from respiration and heart rate without significant loss of purported neuronal-based signal $[9,31]$. Independent component analysis (ICA) was used to identify and remove physiological, movement and thermal (machine) noise components [32, 33], and the six head movement parameters were regressed from the signal.

\section{rsFC and modularity analysis}

To functionally parcellate the rat $\mathrm{mPFC}$, assumed to comprise homologous brain regions to the human ACC [14, 15], we selected voxels that overlapped with $\mathrm{IL}, \mathrm{PrL}$, and cingulate cortices, based on a stereotaxic atlas [34], and conducted a graph-theory based parcellation within this mask. We extracted the time course from each voxel, computed the Pearson correlation between every pair of voxels within the mPFC mask, and correlation coefficients were converted to Fisher's Z-values before applying algebraic and statistical operations. Modularity analysis was conducted using Newman's spectral algorithm for modular detection [35].

To evaluate the FC between the resultant mPFC modules and the striatum in order to recapitulate circuit-nicotine dependence severity relationships observed in human smokers [4-6], the average time course of all voxels within each of the 5 module masks were used as seeds in a subsequent rsFC analysis with the anatomically defined striatum [34] (see Fig. S2). Given the high connectivity between these brain modules (see Fig. S3), we used partial correlations in order to understand the distinct connectivity patterns of our identified functional mPFC modules with the striatum that changed with nicotine dependence severity. The spatial autocorrelation function (ACF), indicating smoothness, was estimated using the $3 \mathrm{dFWHMx}$ function in AFNI for each rat's fMRI image. The ACF computes the spatial autocorrelation of the data. A smoothing parameter which consisted of the averaged ACF across rats was used with the $3 d$ ClustSim function in AFNI to estimate the probability of false positive cluster. The significance threshold was set to $p<0.05$ ( $p_{\text {uncorrected }}<0.01$, cluster size $>0.75 \mathrm{~mm}^{3}$ ). 
a

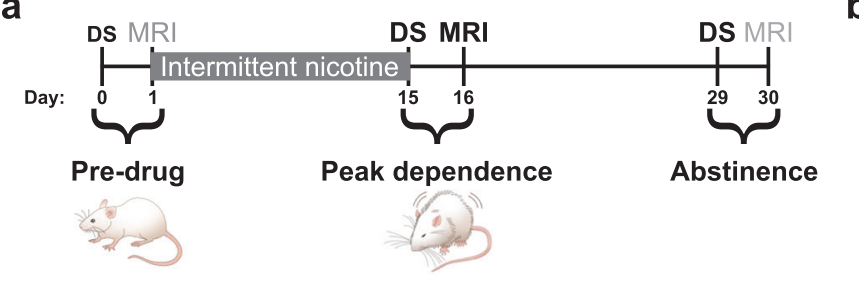

b

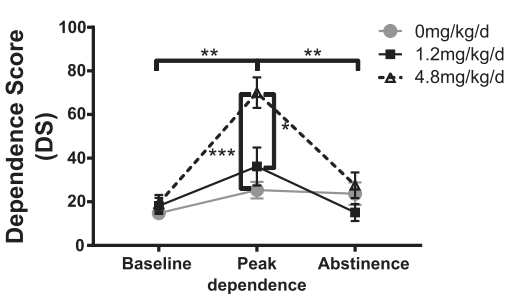

C



Fig. 1 Intermittent nicotine's effects on dependence behavior and the modular definition of the rat mPFC. a Experimental timeline. b Mecamylamine administration (1.5 mg/kg s.c.) induced dose-dependent increase in somatic withdrawal signs (Dependence Score; DS), a marker of nicotine dependence severity, following two weeks of nicotine administration. Exposure to $4.8 \mathrm{mg} / \mathrm{kg} / \mathrm{d}$ of nicotine increased DS compared to baseline and following two weeks abstinence $(p<0.01)$, as well as compared to saline $(p<0.001)$ and $1.2 \mathrm{mg} / \mathrm{kg} / \mathrm{d}$ of nicotine $(p<0.05)$ treated groups. c Modularity analysis of the rat mPFC identified five modules illustrated in 3D color coded composite images. Line graphs depict mean and SEM. ${ }^{*} p<0.05,{ }^{* *} p<0.01,{ }^{* * *} p<0.001$. a, b Adapted from Hsu \& Keeley et al. [10].

Statistics and moderation analysis

A generalized linear mixed-effects (LME) model in MATLAB (MathWorks, Inc.) was used to model the dependence score (the frequency of precipitated somatic withdrawal signs), including fixed effects of nicotine dose and experimental day and their interaction. A second, generalized LME design modeled the effect of FC and nicotine dose on dependence in low and high dose nicotine groups while including the fixed effects of nicotine dose. To assess the association between FC and dependence score, the imaging data on Day 16 (peak nicotine dependence) were used in the model. As a post hoc control, we verified whether the circuits identified at peak dependence, when measured at baseline, were related to dependence behavior at Day 15 (peak dependence) to determine if they were predispositional as well as consequent to nicotine exposure. Separate LME models were used in saline treated rats on all days and in nicotine-exposed rats at pre-drug baseline and post abstinence as a control.

Previous work from our group using the same rat cohorts identified that rsFC between an insular submodule and a striatal module and an insular submodule and a frontal-executive module prior to nicotine administration predicted the subsequent severity of withdrawal signs following chronic nicotine administration [10]. We thus conducted an exploratory moderation analysis to determine whether the relationship between peak withdrawal behavior (our measure of dependence) and FC strength between ACCm-striatum, ILm-NAC and PrLm-NAc was moderated by the FC of these previously determined [10] baseline predictive circuits. A moderation analysis model $[36,37]$ was constructed using the structural equation modeling method [38] in AMOS 17.0. We first identified significant relationships between FC after chronic nicotine administration and nicotine dependence severity. Then, using this moderation model and nicotine-exposed rats $(N=22)$, the FC of both the striatal and frontal-executive submodules at baseline were selected as two moderators of the association between peak dependence FC and withdrawal behavior. In this context, full moderation occurs when the relationship between the independent variable and the dependent variable is no longer significant with the inclusion of a moderator variable [36]. The moderation model was estimated using the maximum likelihood algorithm and statistically tested using a Chi-square test. The moderation effect of each moderator for each brain-behavior relationship was evaluated in the interaction between independent and moderator variables. For significance testing in each path, we utilized permutation testing which randomly resampled the data 100,000 time and estimated the LME model for each permutation. The distribution of $t$-statistic values was obtained, and the significance level was set at $p<0.05$ against the randomized distribution. 
a
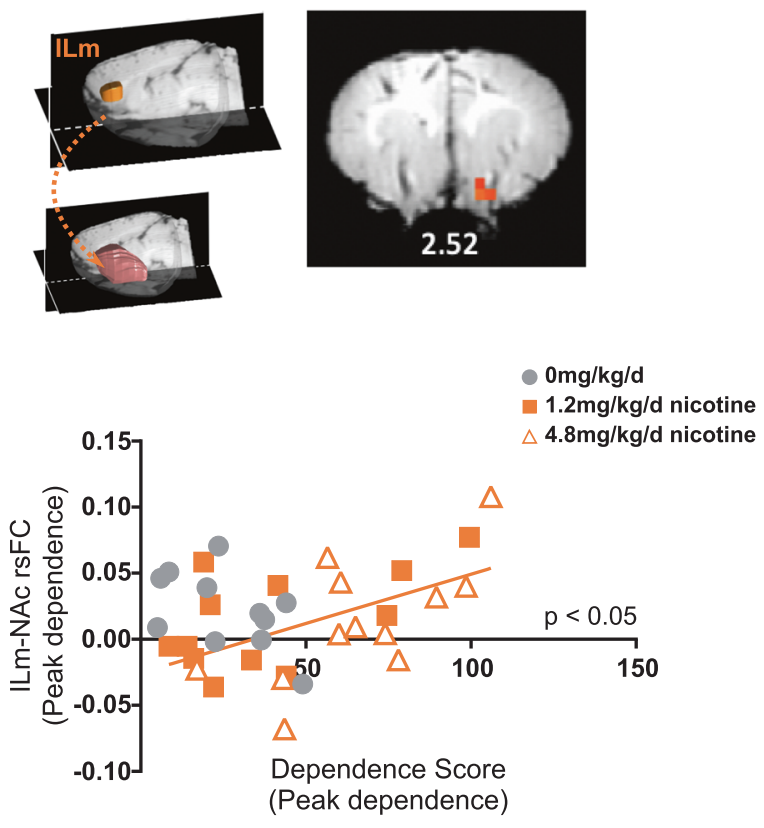

C
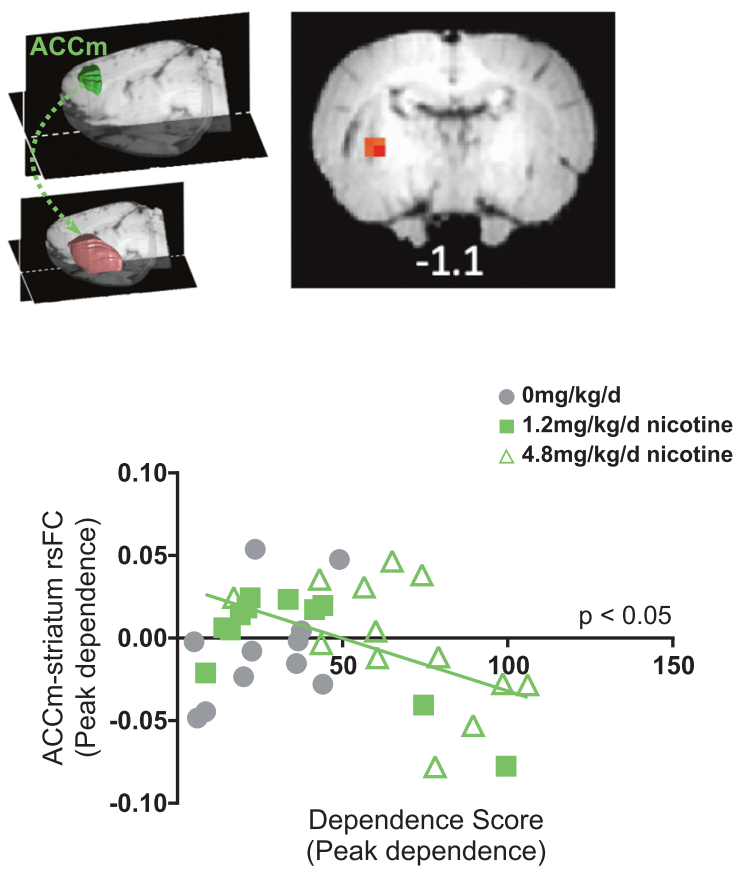

b
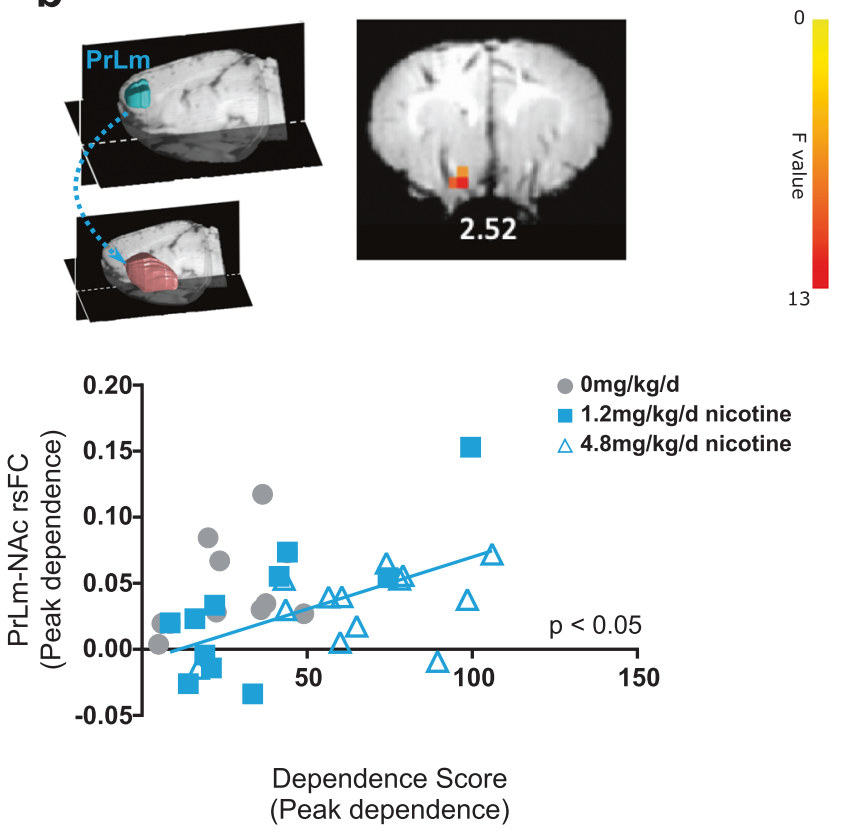

d
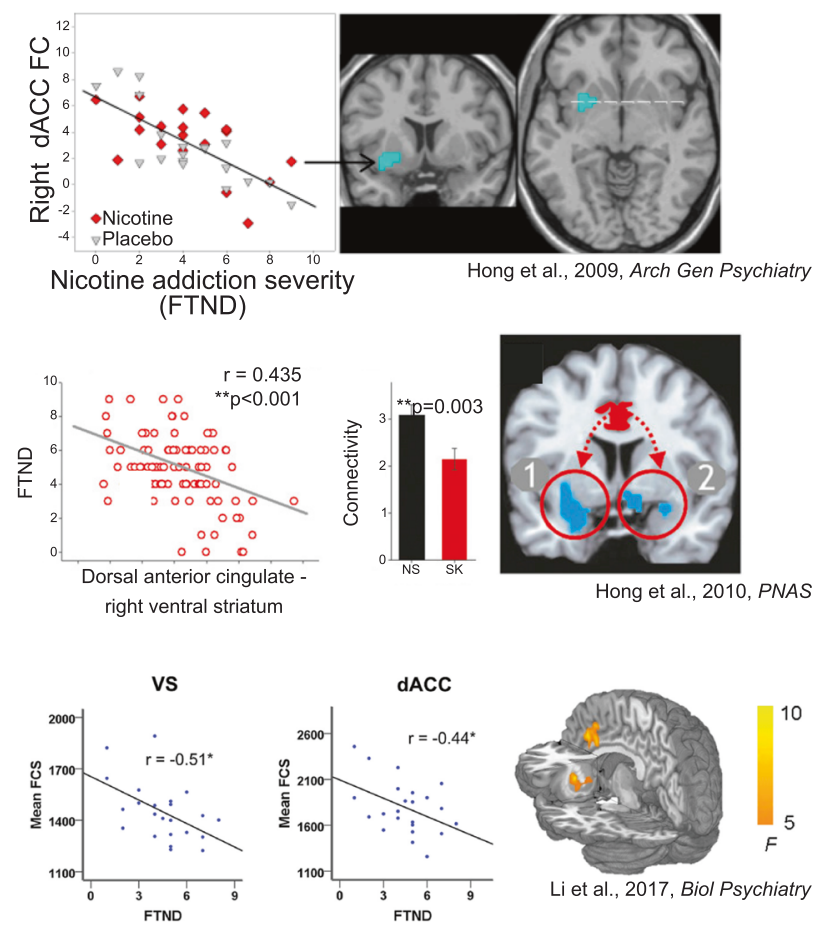

Fig. 2 Anatomical representation of rsFC seeds from three frontal cortical modules ( $a, b$, c; color-coded) to the masked striatum (pink). a The circuit strength between the ILm seed and the right NAc (right panel) was positively correlated $\left(F_{(1,21)}=12.69, p_{\text {corrected }}<0.05\right)$ with dependence severity. $\mathbf{b}$ The circuit strength between the PrLm seed and the left NAc (right panel) was positively associated with increased dependence severity $\left(F_{(1,21)}=15.82, p_{\text {corrected }}<0.05\right)$. c Connectivity strength between the ACCm seed and the left posterior striatum (right panel) was negatively associated with increased nicotine dependence $\left(F_{(1.21)}=13.30, p_{\text {corrected }}<0.05\right)$. $\mathbf{d}$ Circuit exemplars from three human nicotine dependence studies illustrates a negative association between dACC seed and VS (left: top) connectivity and functional connectivity strength (FCS) of dACC and VS (bottom panel) with nicotine dependence severity, as measured with the Fagerstrom Test of Nicotine Dependence (FTND). Each study used different analytical strategies and independent cohorts of smokers and are consistent with the homologous rodent data. Panels adapted from Hong et al. [4], Hong et al. [5], and Li et al. [6]. Note that data from saline-exposed rats (in gray) in panels a-c are graphically represented but were not included in the regression analyses. Numbers under coronal brain sections indicate approximate position from Bregma. 


\section{RESULTS}

When challenged with an acute injection of mecamylamine (1.5 mg/kg s.c.), rats implanted with a modified osmotic minipump (Fig. 1a) demonstrated nicotine dose-dependent increases in dependence severity (Fig. 1b). As reported previously [10], a two-way ANOVA revealed a main effect of Dose $\left(F_{(2,29)}=11.42\right.$, $p<0.001)$ and a Dose $\times$ Day interaction $\left(F_{(4,45)}=8.4, p<0.001\right)$. Peak behavioral withdrawal signs measured at Day 15 returned to baseline levels following 14 days of forced abstinence. Treatment with $4.8 \mathrm{mg} / \mathrm{kg} / \mathrm{d}$ of nicotine increased behavioral withdrawal signs compared to baseline $(p<0.01)$ and following two weeks of abstinence $(p<0.01)$, as well as during peak dependence as compared to treatment with saline $(p<0.001)$ and $1.2 \mathrm{mg} / \mathrm{kg} / \mathrm{d}$ of nicotine $(p<0.05)$.

Modularity analysis of the mPFC resting state BOLD signal collected at peak dependence functionally defined five cortical modules, named here based on their spatial overlap with known neuroanatomical definitions [23]: infralimbic (ILm), prelimbic $(\operatorname{PrLm})$, anterior cingulate $(\mathrm{ACCm})$, middle cingulate and posterior cingulate (Fig. 1C; see Fig. S4 for spatial overlap of modules and neuroanatomy). Using each module as a seed resulted in distinct functional circuit patterns with discrete portions of the striatum (Fig. 1c) that recapitulated known dorsal-ventral and anteriorposterior functional distinctions of the rat mPFC [11-14]. For example, the PrLm connectivity paralleled known anatomical projections to the striatum [11].

We next correlated the strength of the functional circuits at peak dependence between the five prefrontal cortical modules and the striatum (see Fig. S2 for striatal mask) with dependence severity scores at peak dependence to replicate circuit differences that scale with severity of dependence. For this analysis, we used partial correlations in order to understand the distinct connectivity patterns of our identified functional mPFC modules with the striatum that change with nicotine dependence severity, given high connectivity between these modules (see Fig. S3). FC between three of the five modules (PrLm, ILm, and ACCm) and the striatum were significantly related to nicotine dependence severity; no FC-dependence severity relationship was observed for the middle cingulate and posterior cingulate modules, and they were thus not included in any subsequent analyses. Specifically, increased connectivity strength between the ILm and right nucleus accumbens (NAc; $F_{(1,21)}=12.69$, $p_{\text {corrected }}<0.05$; Fig. 2a) and between the PrLm and the left NAC $\left(F_{(1,21)}=15.82, \quad p_{\text {corrected }}<0.05\right.$; Fig. $\left.2 b\right)$, both of which parallel known anatomical projections from IL and PrL to the NAc $[11,39,40]$, were positively associated with dependence severity. There was a significant negative correlation between ACCm and left posterior caudate putamen $\mathrm{rFC}$ and nicotine dependence severity $\left(F_{(1,21)}=13.30, p_{\text {corrected }}<0.05\right.$; Fig. 2 c; referred to ACCm-striatum rsFC for brevity), replicating cross-sectional findings in human smokers (Fig. 2d) [4-6]. Critically, no significant associations between ILm-, PrLm- and ACCm-striatal rsFC and dependence behavior were observed at baseline, post-abstinence (Fig. S5) or at any timepoint among saline-exposed rats (all $p_{\text {corrected }}>0.05$ ). There was no significant relationship between any circuits at baseline and nicotine dependence severity at peak dependence (all $p_{\text {corrected }}>0.05$; Fig. S6). We also observed significant differences between baseline, peak dependence and post abstinence brain-behavior association for the three identified circuits (see Supplemental Results). We conducted simple correlations between the functional modules and the striatum, which revealed the shared variance in connectivity between MPFC modules and the striatum that changed as a function of nicotine dependence severity. Notably, simple correlations revealed similar significance maps between all of the identified mPFC modules and the striatum, with the exception of the posterior cingulate seed (see Fig. S7). All $p$-values were Bonferroni corrected across 3 seeds, 3 days and 2 treatment conditions (saline vs nicotine). It is important to note that the rsFC of these circuits did not change as a function of nicotine exposure (Fig. S8). These results suggest that the relationships between frontal cortico-striatal connectivity and nicotine dependence are consequent to chronic nicotine exposure.

We next sought to determine whether these nicotine dependent mPFC-striatal circuit changes were influenced by circuits that predispose an animal to dependence [10]. Path analysis, using nicotine-exposed rats $(N=22)$, identified a significant relationship between nicotine dependent-changes in $\mathrm{MPFC}$-striatal circuits at peak dependence (Chi-square $=7.9, \mathrm{df}=3, p=0.048$ ). With the inclusion of pre-drug insular-striatal and insular-frontal-executive connectivity strength as moderators, a significant moderation model was identified (Chi-square $=133.756, \mathrm{df}=27, p \leq 0.001$ ). Path analysis demonstrated a significant impact of two circuits: ILm-NAc FC (path coefficient $=0.514, p<0.05$ ) and PrLm-NAc FC (path coefficient $=0.78, p<0.01$ ) on nicotine dependence severity at peak dependence. Both the relationship between ILm(interaction coefficient $=0.822, p<0.05$ ) and PrLm-NAc rsFC (interaction coefficient $=-0.856, p<0.01$ ) and nicotine dependence severity were partially moderated by pre-drug, baseline insular-frontal-executive rsFC. Notably, the relationship between $\mathrm{ACCm}$-striatal rsFC and dependence severity at peak dependence (path coefficient $=0.108$, n.s.) was fully moderated by pre-drug, baseline insular-frontal/executive rsFC (interaction effect $=0.827$, $p<0.05$ ) and baseline insular-striatal rsFC (interaction effect = $-0.706, p<0.05$; Fig. 3). Importantly, full moderation was unique to this circuit (Fig. S9). In sum, changes in ACCm-striatal circuit strength, which tracks nicotine-induced dependence, are moderated by individual differences in predispositional insular-frontal/ executive circuit and insular-striatal circuit strength.

\section{DISCUSSION}

Convergent with extant literature using independent smoking cohorts and distinct analytic strategies [4-6], we observed a decrease in ACC-striatal FC with increasing nicotine-induced dependence severity. Using data-driven modularity to parse the rat prefrontal cortex into functional units, we identified a circuit between the ACCm (comprising portions of PrL and Cg1 [34]) and the left posterior caudate, that negatively scaled with dependence severity; this effect was not observed for FC between other mPFC modules (discussed below) and was not observed as a predictor circuit at baseline. The striatum and ACC, which are activated by nicotine in humans and rodents [41, 42], are integral nodes in circuits implicated in substance use disorders [43]. Allelic variation in nicotine metabolism in humans, resulting in a presumptive increase in brain nicotine concentration [44], is associated with decreased network hub connectivity strength in both dACC and VS and, in turn, with increased nicotine dependence [6], supporting the idea that changes in ACC-striatal connectivity are consequential to chronic, dependence producing nicotine exposure. Our data are convergent with known changes in BOLD signal response and rsFC between prefrontal and striatal circuits in rats with intermittent and repeated doses of nicotine [45-47]. Taken together with other preclinical and human research, the present longitudinal data suggest that the ACC-striatal circuit identified herein, which parallel cross-sectional findings in human smokers [4-6], is not predispositional [10], but rather is a nicotineinduced trait marker of nicotine dependence [3]. Finally, this work provides evidence that the rat $A C C m$ is a/the putative functional homologue of human $\mathrm{AACC}$, as it follows a consistent directional change in connectivity with the development of nicotine dependence.

The identified functional ACCm-striatal circuit relationship with nicotine dependence severity critically builds upon our recently identified pre-drug exposure insular-frontal circuit as a predictor of subsequent nicotine dependence severity [10]. Convergent with the role of the insula, our previous cross-sectional human smoker studies identified the insula as critical for the interaction between 




Fig. 3 Graphic illustration of the moderation analysis. Results depict the relationship between nicotine dependence severity (dependent variable) and mPFC- (ILm, PrLm and ACCm) striatal circuits from Fig. 2 (independent variables) with the influence (moderation) of predrug insular-striatal and insular -frontal-executive circuits (moderators). The baseline moderators were derived from previously published findings (Hsu et al. [10]). Path analysis using only nicotine-exposed rats $(N=22)$ identified a significant relationship between nicotine dependent-changes in MPFC-striatal circuits at peak dependence. The association between PrLm- and ILm-NAc rsFC and dependence severity were partially moderated whereas the association between ACCm-striatal rsFC and baseline dependence severity was fully moderated by baseline insular-frontal-executive rsFC and baseline insular-striatal rsFC. Dotted lines between brain ROIs represent rsFC. Solid lines indicate significant moderation, dashed lines represent non-significant moderation effects and dotted lines represent rsFC. Arrowheads of dotted lines (i.e. rsFC) do not denote directionality and only indicate the seed and consequent rsFC results. n.s. $p>0.05,{ }^{*} p<0.05,{ }^{* *} p<0.01$.

nicotine metabolism-induced dACC and ventral striatal connectivity hub strength [6]. The insula and ACC are key nodes in the Salience Network (SN) [48], and the balance between the SN and networks driving self-referential thought and executive control are hypothesized as key to allocating cognitive resources in health and neuropsychiatric disease $[49,50]$, including nicotine dependence [51]. Herein, using an exploratory moderation analysis, we demonstrate that the relationship between dependence severity and ACCm-striatal FC strength is statistically moderated by predispositional individual differences in connectivity between insular and both frontal-executive regions, which overlap with the identified $A C C m$, and the striatum. Thus, changes in the association between ACC-striatal connectivity and nicotine dependence behavior following nicotine administration predominantly occurs in those individuals with increased baseline insularfrontal and insular-striatal circuit connectivity. In other words, we provide evidence that insular-ACC and insular-striatal connectivity strength may bias downstream ACC-striatal connectivity and its relationship to severity of dependence following exposure to nicotine, and this effect is observed particularly within this apparent homologous circuit in rodents and humans.

These findings provide evidence for the rat $A C C m$ as a/the putative functional homologue of human dACC, in agreement with anatomical and structural connectivity analyses across species [11, 40,52], since its connectivity with the striatum is also negatively associated with nicotine dependence severity. Here, ACCm-left posterior caudate connectivity was associated with nicotine dependence severity as measured with somatic signs of withdrawal whereas in our previous human studies, ACCventral striatum connectivity was associated with the severity of nicotine dependence as indexed by the behavioral scores on the Fagerstrom Test for Nicotine Dependence [4-6]. In those studies, while the cluster identified in the striatum was named 'ventral striatum,' this statistically significant group of voxels, across these three studies, included other regions which overlapped with portions of the dorsal striatum (see Fig. 2d), as observed herein. Any regions that fall outside of this overlap may be a result of species differences across humans and rodents and/or the different measures of dependence estimation. Furthermore, the observed negative association between ACCm-striatum and nicotine dependence severity was observed using partial correlations, which can identify the unique contributions of each module to the relationship between striatal connectivity and nicotine dependence severity. Notably, simple correlation analysis revealed overlap in the pattern of connectivity with the striatum that varied as a function of nicotine dependence, further supporting the use of partial correlations to reveal distinct functional connectivity patterns that can parallel known anatomical connections [53]. Given the known functional parcellations of the rat mPFC [11] and their separate roles in substance use disorders [43], the identification of unique (using partial correlations) as compared to common (using simple correlations) mPFC module-striatal connectivity best addressed the question of recapitulating, in rodents, a circuit first described in human smokers. 
We also demonstrated functional distinctions between $\mathrm{PrL}$, IL, and the Cg1/2 subregions [11, 14, 54]. PrLm-NAC and ILm-NAC connectivity shared consistent positive relationships with dependence, although both were only partially moderated by baseline insular connectivity. $\mathrm{PrL}$ and $\mathrm{IL}$ share extensive reciprocal connectivity [54], both project to the NAc [11,39,40] and are implicated in the etiology of substance use disorders [43]. The positive association between ILm- and PrLm-NAc connectivity and dependence severity may reflect the role of these circuits in aspects of nicotine dependence outside of the currently measured somatic signs of dependence severity, which represents only one facet of the complex, multi-dimensional nicotine withdrawal syndrome [55-58]. For example, that smokers show enhanced striatal-PFC FC strength following smoking cue presentation [59] and IL and PrL connectivity with the striatum increases following acute and chronic administration of nicotine [45-47] may help explain the greater connectivity strength between frontal-striatal regions observed here. Future work should seek to identify the relationship between the IL- and PrL-striatal connectivity with nicotine dependence and nicotine withdrawal syndrome, including changes in affective and executive functions [60].

Despite the strengths of the current study, several limitations remain. Notably, these data were previously analyzed to identify circuits that predict nicotine dependence severity [10]. Replicating these findings and those observed here in an independent sample would further strengthen and support these observations. Furthermore, MRI data acquisition during peak dependence could have been influenced by acute, spontaneous withdrawal, given that the modified osmotic minipumps were formulated to last for 14 days of nicotine administration. Although there was no change in anesthesia plane or any physiological measure reflective of withdrawal as a function of nicotine administration and scanning days (see Fig. S1), this does not necessarily preclude an interaction between nicotine withdrawal and anesthesia that might have contributed to these correlations. Finally, although statistically significant, our moderation analysis should be considered exploratory as we had available a relatively small sample size $(N=22)$ which may have led to an underpowered analysis model.

These limitations notwithstanding, we interpret our findings that a pre-drug administration insular-ACC functional circuit within a rodent homologue of the human $\mathrm{SN}$, first observed crosssectionally in humans and recapitulated herein, may drive nicotine-induced changes in ACC-striatal connectivity and its relationship with nicotine dependence severity. Our findings describe the intersection between predispositional individual differences and drug-induced causation. Since decreased connectivity between ACC and the striatum has also been associated with predicting relapse following a quit attempt [61], this circuit may serve as a novel treatment target. Directly targeting this cingulate-striatal circuit, whether with optogenetic, chemogenetic or noninvasive brain stimulation, may help determine whether such a circuit-based approach is a clinically useful treatment biomarker that could increase the probability to successfully quit smoking and thus reduce disease burden.

\section{FUNDING AND DISCLOSURE}

Supported by the Intramural Research Program of NIDA/NIH, FDA Center on Tobacco Products (grant \# NDA 13001-001-00000 to EAS) and a postdoctoral fellowship award from the Canadian Institutes of Health Research (CIHR) to RJK (FRN 152478). The authors have declared that no conflict of interest exists.

\section{ACKNOWLEDGEMENTS}

Special thanks to Marc Raley (NIDA-IRP) for help with illustrations.

\section{ADDITIONAL INFORMATION}

Supplementary Information accompanies this paper at (https://doi.org/10.1038/ s41386-020-0635-x).

Publisher's note Springer Nature remains neutral with regard to jurisdictional claims in published maps and institutional affiliations.

\section{REFERENCES}

1. Surgeon General. The Health Consequences of Smoking - 50 Years of Progress: A Report of the Surgeon General, 2014. (2014). http://www.surgeongeneral.gov/ library/reports/50-years-of-progress/index.html\#fullreport. Accessed 31 Aug 2015.

2. Centers for Disease Control and Prevention (US), National Center for Chronic Disease Prevention and Health Promotion (US), Office on Smoking and Health (US). How Tobacco Smoke Causes Disease: The Biology and Behavioral Basis for Smoking-Attributable Disease: A Report of the Surgeon General. Atlanta (GA): Centers for Disease Control and Prevention (US); 2010.

3. Sutherland MT, Stein EA. Functional neurocircuits and neuroimaging biomarkers of tobacco use disorder. Trends Mol Med. 2018:24:129-43.

4. Hong LE, Gu H, Yang Y, Ross TJ, Salmeron BJ, Buchholz B, et al. Association of nicotine addiction and nicotine's actions with separate cingulate cortex functional circuits. Arch Gen Psychiatry. 2009;66:431-41.

5. Hong LE, Hodgkinson CA, Yang Y, Sampath H, Ross TJ, Buchholz B, et al. A genetically modulated, intrinsic cingulate circuit supports human nicotine addiction. Proc Natl Acad Sci USA. 2010;107:13509-14.

6. Li S, Yang Y, Hoffmann E, Tyndale RF, Stein EA. CYP2A6 genetic variation alters striatal-cingulate circuits, network hubs, and executive processing in smokers. Biol Psychiatry. 2017;81:554-63.

7. Sumiyoshi A, Keeley RJ, Lu H. Physiological Considerations of Functional Magnetic Resonance Imaging in Animal Models. Biol Psychiatry Cogn Neurosci Neuroimaging. 2018. https://doi.org/10.1016/j.bpsc.2018.08.002. Accessed 17 Aug 2018.

8. Lu H, Zou Q, Gu H, Raichle ME, Stein EA, Yang Y. Rat brains also have a default mode network. Proc Natl Acad Sci USA. 2012;109:3979-84.

9. Hsu L-M, Liang X, Gu H, Brynildsen JK, Stark JA, Ash JA, et al. Constituents and functional implications of the rat default mode network. Proc Natl Acad Sci USA. 2016;113:E4541-7.

10. Hsu L-M, Keeley RJ, Liang X, Brynildsen JK, Lu H, Yang Y, et al. Intrinsic insularfrontal networks predict future nicotine dependence severity. J Neurosci. 2019. https://doi.org/10.1523/JNEUROSCI.0140-19.2019. Accessed 16 Apr 2019.

11. Heilbronner SR, Rodriguez-Romaguera J, Quirk GJ, Groenewegen HJ, Haber SN. Circuit-based corticostriatal homologies between rat and primate. Biol Psychiatry. 2016;80:509-21.

12. Heidbreder CA, Groenewegen HJ. The medial prefrontal cortex in the rat: evidence for a dorso-ventral distinction based upon functional and anatomical characteristics. Neurosci Biobehav Rev. 2003;27:555-79.

13. Hamilton DA, Brigman JL. Behavioral flexibility in rats and mice: contributions of distinct frontocortical regions. Genes Brain Behav. 2015;14:4-21.

14. Laubach M, Amarante LM, Swanson K, White SR. What, if anything, is rodent prefrontal cortex? ENeuro. 2018;5:0315-18.

15. Paxinos G, Watson C. The Rat brain in stereotaxic coordinates: hard cover edition. Elsevier Science; 2013.

16. Maslov S, Sneppen K. Specificity and stability in topology of protein networks. Science. 2002;296:910-3.

17. Brynildsen JK, Najar J, Hsu L-M, Vaupel DB, Lu H, Ross TJ, et al. A novel method to induce nicotine dependence by intermittent drug delivery using osmotic minipumps. Pharmacol Biochem Behav. 2016. https://doi.org/10.1016/j.pbb.2015.12.010. Accessed 2 Jan 2016.

18. Hughes JR. Effects of abstinence from tobacco: etiology, animal models, epidemiology, and significance: a subjective review. Nicotine Tob Res. 2007;9:329-39.

19. Hughes JR. Effects of abstinence from tobacco: valid symptoms and time course. Nicotine Tob Res. 2007;9:315-27.

20. Piasecki TM, Niaura R, Shadel WG, Abrams D, Goldstein M, Fiore MC, et al. Smoking withdrawal dynamics in unaided quitters. J Abnorm Psychol. 2000;109:74-86.

21. Piasecki TM, Fiore MC, Baker TB. Profiles in discouragement: two studies of variability in the time course of smoking withdrawal symptoms. J Abnorm Psychol. 1998;107:238-51.

22. Malin DH, Lake JR, Newlin-Maultsby P, Roberts LK, Lanier JG, Carter VA, et al. Rodent model of nicotine abstinence syndrome. Pharm Biochem Behav. 1992;43:779-84.

23. Cross SJ, Linker KE, Leslie FM. Sex-dependent effects of nicotine on the developing brain. J Neurosci Res. 2017;95:422-36. 
24. Benwell ME, Balfour DJ, Birrell CE. Desensitization of the nicotine-induced mesolimbic dopamine responses during constant infusion with nicotine. $\mathrm{Br} J$ Pharm. 1995;114:454-60.

25. Watkins SS, Stinus L, Koob GF, Markou A. Reward and somatic changes during precipitated nicotine withdrawal in rats: centrally and peripherally mediated effects. J Pharm Exp Ther. 2000;292:1053-64.

26. Vann RE, Balster RL, Beardsley PM. Dose, duration, and pattern of nicotine administration as determinants of behavioral dependence in rats. Psychopharmacology. 2006;184:482-93.

27. Brynildsen JK, Hsu LM, Ross TJ, Stein EA, Yang Y, Lu H. Physiological characterization of a robust survival rodent fMRI method. Magn Reson Imaging. 2017;35:54-60.

28. Lu H, Stein EA. Resting state functional connectivity: its physiological basis and application in neuropharmacology. Neuropharmacology. 2014;84:79-89.

29. Cox RW. AFNI: software for analysis and visualization of functional magnetic resonance neuroimages. Comput Biomed Res. 1996;29:162-73.

30. Lu H, Scholl CA, Zuo Y, Demny S, Rea W, Stein EA, et al. Registering and analyzing rat $\mathrm{fMRI}$ data in the stereotaxic framework by exploiting intrinsic anatomical features. Magn Reson Imaging. 2010;28:146-52.

31. Kalthoff D, Seehafer JU, Po C, Wiedermann D, Hoehn M. Functional connectivity in the rat at $11.7 \mathrm{~T}$ : Impact of physiological noise in resting state fMRI. Neuroimage. 2011;54:2828-39.

32. Griffanti L, Douaud G, Bijsterbosch J, Evangelisti S, Alfaro-Almagro F, Glasser MF, et al. Hand classification of fMRI ICA noise components. Neuroimage. 2017;154:188-205.

33. Rummel C, Verma RK, Schöpf V, Hauf M, Abela E, Zapata Berruecos JF, et al. Time course based artifact identification for independent components of resting-state fMRI. Front Hum Neurosci. 2013;7:214.

34. Paxinos G, Watson C. The rat brain in stereotaxic coordinates. Academic press; 2007.

35. Newman MEJ. Modularity and community structure in networks. Proc Natl Acad Sci USA. 2006;103:8577-82.

36. Baron RM, Kenny DA. The moderator-mediator variable distinction in social psychological research: conceptual, strategic, and statistical considerations. J Pers Soc Psychol. 1986;51:1173-82.

37. Kenny DA, Kashy DA, Bolger N. Data analysis in social psychology. Handb Soc Psychol. 1998;1:233-65.

38. Boucard A, Marchand A, Noguès X. Reliability and validity of structural equation modeling applied to neuroimaging data: a simulation study. J Neurosci Methods. 2007; 166:278-92.

39. Voorn P, Vanderschuren LJMJ, Groenewegen HJ, Robbins TW, Pennartz CMA. Putting a spin on the dorsal-ventral divide of the striatum. Trends Neurosci. 2004;27:468-74.

40. Vogt BA, Paxinos G. Cytoarchitecture of mouse and rat cingulate cortex with human homologies. Brain Struct Funct. 2014;219:185-92.

41. Stein EA, Pankiewicz J, Harsch HH, Cho JK, Fuller SA, Hoffmann RG, et al. Nicotineinduced limbic cortical activation in the human brain: a functional MRI study. Am J Psychiatry. 1998:155:1009-15.

42. Grünwald F, Schröck $H$, Kuschinsky W. The effect of an acute nicotine infusion on the local cerebral glucose utilization of the awake rat. Klin Wochenschr. 1988;66 (Suppl 11):37-41.
43. Koob GF, Volkow ND. Neurobiology of addiction: a neurocircuitry analysis. Lancet Psychiatry. 2016:3:760-73.

44. Ferguson CS, Tyndale RF. Cytochrome P450 enzymes in the brain: emerging evidence of biological significance. Trends Pharm Sci. 2011;32:708-14.

45. Huang W, Tam K, Fernando J, Heffernan M, King J, DiFranza JR. Nicotine and resting-state functional connectivity: effects of intermittent doses. Nicotine Tob Res. 2015;17:1311-7.

46. King J, Huang W, Chen W, Heffernan M, Shields J, Rane P, et al. A comparison of brain and behavioral effects of varenicline and nicotine in rats. Behavioural Brain Res. 2011;223:42-47.

47. Li Z, DiFranza JR, Wellman RJ, Kulkarni P, King JA. Imaging brain activation in nicotine-sensitized rats. Brain Res. 2008;1199:91-99.

48. Seeley WW, Menon V, Schatzberg AF, Keller J, Glover GH, Kenna H, et al. Dissociable intrinsic connectivity networks for salience processing and executive control. J Neurosci. 2007;27:2349-56.

49. Menon V, Uddin LQ. Saliency, switching, attention and control: a network mode of insula function. Brain Struct Funct. 2010;214:655-67.

50. Uddin LQ, Supekar K, Menon V. Typical and atypical development of functional human brain networks: insights from resting-state FMRI. Front Syst Neurosci. 2010;4:21.

51. Sutherland M, Mchugh M, Pariyadath V, Stein E. Resting state functional connectivity in addiction: lessons learned and a road ahead. Neuroimage. 2012;62:2281-95.

52. Vogt BA, Hof PR, Zilles K, Vogt LJ, Herold C, Palomero-Gallagher N. Cingulate area 32 homologies in mouse, rat, macaque and human: cytoarchitecture and receptor architecture. J Comp Neurol. 2013;521:4189-204.

53. Caparelli EC, Ross TJ, Gu H, Liang X, Stein EA, Yang Y. Graph theory reveals amygdala modules consistent with its anatomical subdivisions. Sci Rep. 2017;7:14392

54. Ito HT, Zhang S-J, Witter MP, Moser El, Moser M-B. A prefrontal-thalamohippocampal circuit for goal-directed spatial navigation. Nature. 2015;522:50-55.

55. Bauzo RM, Bruijnzeel AW. Animal models of nicotine withdrawal: intracranial selfstimulation and somatic signs of withdrawal. Methods Mol Biol. 2012;829:257-68.

56. De Biasi M, Dani JA. Reward, addiction, withdrawal to nicotine. Annu Rev Neurosci. 2011;34:105-30.

57. Kenny PJ, Markou A. Neurobiology of the nicotine withdrawal syndrome. Pharm Biochem Behav. 2001;70:531-49.

58. McLaughlin I, Dani JA, De Biasi M. Nicotine withdrawal. Curr Top Behav Neurosci. 2015;24:99-123.

59. Janes AC, Nickerson LD, Frederick BDB, Kaufman MJ. Prefrontal and limbic resting state brain network functional connectivity differs between nicotinedependent smokers and non-smoking controls. Drug Alcohol Depend. 2012;125:252-9.

60. Levin ED, Torry D. Acute and chronic nicotine effects on working memory in aged rats. Psychopharmacology. 1996;123:88-97.

61. Sweitzer MM, Geier CF, Addicott MA, Denlinger R, Raiff BR, Dallery J, et al Smoking abstinence-induced changes in resting state functional connectivity with ventral striatum predict lapse during a quit attempt. Neuropsychopharmacology. 2016;41:2521-9. 